\title{
Lung CT Image Recognition using Deep Learning Techniques to Detect Lung Cancer
}

\author{
Prathyusha Chalasani ${ }^{1}, \mathrm{~S}$ Rajesh ${ }^{2}$ \\ ${ }^{1}$ Department Of CSE,VR Siddhartha Engineering College,Vijayawada,India,prathyusha020@ gmail.com \\ ${ }^{2}$ Department Of CSE,VR Siddhartha Engineering College,Vijayawada,India,rssingam99@ gmail.com
}

\begin{abstract}
Now a days, The digital image recognition techniques are extensively used in a couple of therapeutic domains for image improvement in earlier division and treatment stages, where the time factor is basic to discover the variety from the standard issues in target pictures, especially in various threatening development tumors, for instance, and lung infection. Lung cancer diagnosis is the most important early-stage research field for researcher. The proposed system is designed for the premature detection of lung cancer in two stages. The proposed method consists of several steps such as image acquisition, preprocessing, trivialization, thresholding, segmentation, extraction of features, and detection of neural networks. At first, the lung CT images they're input into the system and then passed through the preprocessing phase of the image using some processing techniques. Lung cancer growth is the most significant sickness cause high death rate. Also, computer vision methods helped determination can be valuable for doctors to precisely recognize the malignant growth cells. Numerous computer vision supported strategies have been contemplated and applied utilizing image recognition and deep learning. To minimize their effect in classification, the JSRT data set is considered to be the most commonly used reference data set to perform experiments. The right segmentation of the lung tumor from X-rays, CT-scans or MRI are the steps towards an integrated diagnostic device for the detection of lung cancer To train this neural network using volumes with tumor size and position, our detection is. In recent techniques, such as machine learning or deep learning, lung cancer can be predicted but this technique is not appropriate for predicting image segmentation in that particular field.
\end{abstract}

Key words : : Lung Cancer, CT scan, X-Ray, JSRT Dataset, MRI, Detection, Deep Learning, Acquisition, Segmentation.

\section{INTRODUCTION}

Cancer is the major terminal illness globally, causing an estimated 9.6 million deaths in 2018. Of several forms of cancer, lung cancer is one of the prevalent death-causing diseases and is found in both genders with an estimated death of 1.76 million in 2018.
The unbounded development of atypical cells that are not typical in one or the two lungs is Lung malignant growth. These irregular cells disturb the elements of ordinary lung cells which may prompt an unfortunate tissue in lung. The development of these unnatural advancements leads to the formation and emergence of tumors, and disturbs the lung usability that supplies the body with oxygen through blood. In the screening of the lungs, computed tomography (CT) has outperformed conventional radiography because it generates very detailed high-resolution images and can show early-stage lesions that are too small to be detected CT has been generally used to recognize various lung infections, including pneumoconiosis, pneumonia, aspiratory edema, and lung malignancy [1]. Early discovery of illnesses is vital for treatment arranging. Be that as it may, it is vietheyd as one of the most testing undertakings performed by radiologists because of the enormous measure of information produced by CT filter. Along these lines, PC helped symptomatic (CAD) frameworks are expected to help radiologists in the examination and assessment of CT checks. A CAD framework dissects clinical pictures in a few stages: initial a preprocessing step for commotion decrease and improving the picture quality and afterward division venture to separate area of intrigue (ROI) from different structures in the picture. After division, various highlights, for example, geometrical, textural, and factual highlights are extricated. At long last, a grouping/assessment step is done to assess and analyze the ROI dependent on separated highlights.

\section{STUDY AREA}

In this section, they discuss on different existing studies was proposed by different authors regarding on lung CT image classification using deep learning models.

Pietro Nardelli et.al, [1] presented a technique titled as Pulmonary Artery-Vein Classification in CT Images Using Deep Learning. In this work, they present a novel, completely programmed way to deal with grouping vessels from chest CT pictures into courses and veins. The calculation follows three principle steps: initial, a scale-space particles division to disconnect vessels; at that point a 3D convolutional neural system $(\mathrm{CNN})$ to acquire a first order of vessels; at long last, diagram cuts (GC) improvement to refine the outcomes. Yutong Xie et.al, [2] Knowledge-based Collaborative Deep Learning for Benign-Malignant Lung Nodule Classification on Chest CT. In this paper, they propose a multi-see 
information based collective (MV-KBC) profound model to isolate threatening from amiable knobs utilizing constrained chest CT information. Our model learns 3D lung knob attributes by breaking down a 3D knob into nine fixed perspectives.

Ashnil Kumar et.al, [3] introduced a strategy titled as Co-Learning Feature Fusion Maps from PET-CT Images of Lung Cancer. They assessed the capacity of our CNN to distinguish and fragment numerous locales with various combination necessities utilizing a dataset of PET-CT pictures of lung malignancy. They contrasted our strategy with benchmark methods for multi-methodology picture combination procedures, and multichannel (MC) strategies) and division. These strategies for the most part don't consider the spatially changing visual qualities that encode diverse data over the various modalities, which have various needs at various areas. QINGFENG WANG et.al, [4] WGAN-Based Synthetic Minority Over-Sampling Technique: Improving Semantic Fine-Grained Classification for Lung Nodules in CT Images. In this paper, the WGAN based over-examining strategy is applied to expand the information to adjust for the fine-grained characterization of seven semantic qualities of lung knobs in figured tomography pictures. The fine-grained arrangement is completed with a typical convolutional neural system (CNN). TAO HAN et.al, [5] Internet of Medical Things-Based on Deep Learning Techniques for Segmentation of Lung and Stroke Regions in CT Scans. In this work, they propose a technique dependent on the soundness of things for the arrangement and division of CT pictures of the lung and hemorrhagic stroke. The framework called HTSCS - Medical Images: Health-of-Things System for the Classification and Segmentation of Medical Images, utilizes move learning between models dependent on profound learning joined with old style techniques for calibrating. The proposed strategy acquired fantastic outcomes for the grouping of hemorrhagic stroke and aspiratory districts, with estimations of up to $100 \%$ exactness.

Heewon Chung et.al, [6] Automatic Lung Segmentation with Juxta-Pleural Nodule Identification utilizing Active Contour Model and Bayesian Approach. They propose a novel lung division strategy to limit the juxta-pleural knob issue, an infamous test in the applications. Technique: They at first utilized the Chan-Vese (CV) model for dynamic lung forms, and embraced a Bayesian methodology dependent on the $\mathrm{CV}$ model outcomes, which predicts the lung picture dependent on the portioned lung shape in the past edge picture or neighboring upper casing picture. Xiaomei Li et.al, [7] presented a paper called as Knockoff channel based component choice for segregation of non-little cell lung malignant growth in CT picture. It stays an extreme errand since different picture highlights could be extricated from one single picture while some portion of the highlights is unessential to the last conclusion results.

In this investigation, a knockoff channel based methodology is proposed to create the ideal list of capabilities and to limit the insignificance of the yield highlights for the grouping of lung malignant growth in CT pictures.
Receptacle WANG et.al, [8] A Fast and Efficient CAD System for Improving the Performance of Malignancy Level Classification on Lung Nodules. In this investigation, they present a quick and proficient CAD framework to improve the exhibition of knobs danger level characterization. Right off the bat, to decrease bogus positives (FPs), they propose a novel vessel division technique which estimates vessel probability by cylindrical like structures separating from numerous perspectives. Retz Mahima Devarapalli et.al, [9] introduced a paper titled as Lung Cancer Detection of CT Lung Images. To recognize disease different picture handling procedures have been advanced and applied like middle wiener channel in the preprocessing stage. Sarah E. Gerard et.al, [10] Fissure Net: A Deep Learning Approach for Pulmonary Fissure Detection in CT Images. The proposed system, called Fissure Net, is a coarse-to-fine course of two convolutional neural systems. The coarse-to-fine system reduces the difficulties related with preparing a system to fragment a slight structure that speaks to a little part of the picture voxels.

\section{MATERIALS AND METHODS}

The system by which lung CT images are identified using deep neural networks to detect lung cancer, as shown in figure 1.

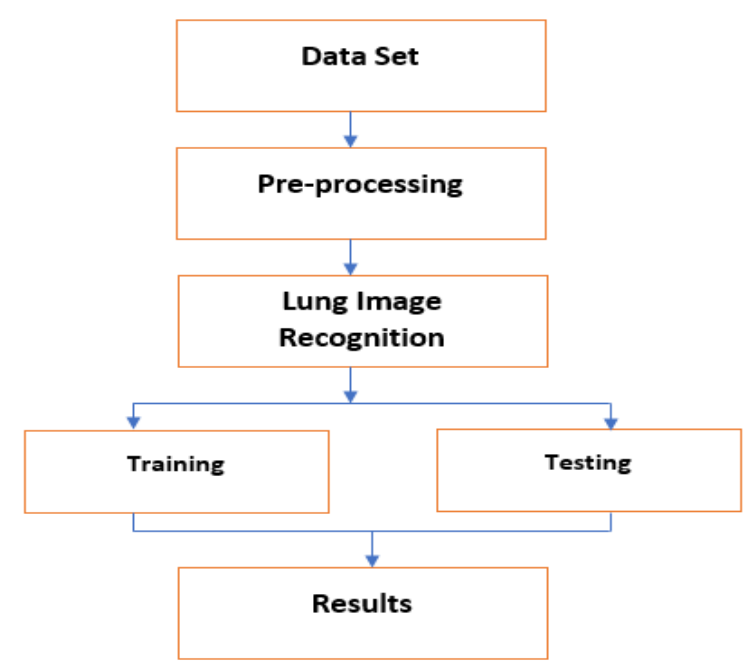

Figure 1: Architecture of our proposed system.

The proposed method comprises of multiple modules used by deep learning models to classify CT images of the lung. They are, do they?
- Data Set
- Pre-processing
- Lung Image recognition
- Model Training
- Model Testing 


\subsection{Data Set}

In this technique, our data set, called as JSRT image data set, contains 247 images of 154 cases with lung nodules and 93 cases without lung nodules. BSE-JSRT data set includes 247 images of the JSRT data set, but no clavicle and rib shadows are eliminated with the special algorithms. We applied classification techniques for this collection of data, respectively. The database contains the following information about each patient in a text file: nodule size, age, gender, final diagnosis, location of the anatomic nodule, $\mathrm{x}-$ and $y$-coordinates of the 20 nodule centers in a digital image. And, the sample images of the given set of data are shown in figure 2 below.

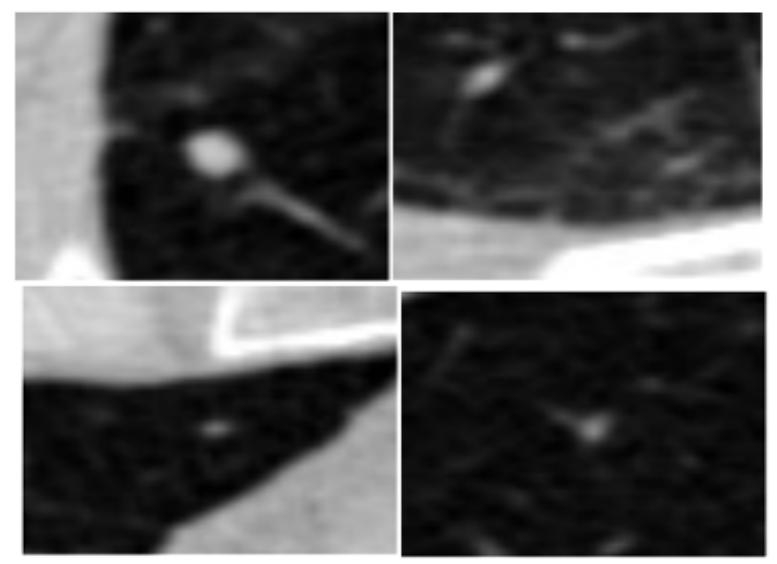

Figure 2: Sample Image Data set as input.

\subsection{Image Pre-processing}

Preprocessing step is aimed at removing unwanted noise and enhancing the image quality. We used a median filtering to remove noise whilst maintaining the contours and good lung details.

\subsection{Lung Image recognition}

Machine learning techniques like vector supporting devices are often used for the identification and recognition of tumors in this step. But, when we define features, the assumptions we make often limit them. It leads to diminished sensitivity. However, deep learning might be an optimal option, since these techniques can understand features from raw image data. One difficulty in applying such methodologies is the lack of labeled medical image data. While it is a disadvantage with all deep learning techniques, due to privacy issues with patients, it's more so for medical image data. In this, a network of neural convolution will be developed, equipped, and detected lung nodules. We are using the data from the Lung Image Database Consortium and Infectious Disease Research Initial step was to create an image database for training.

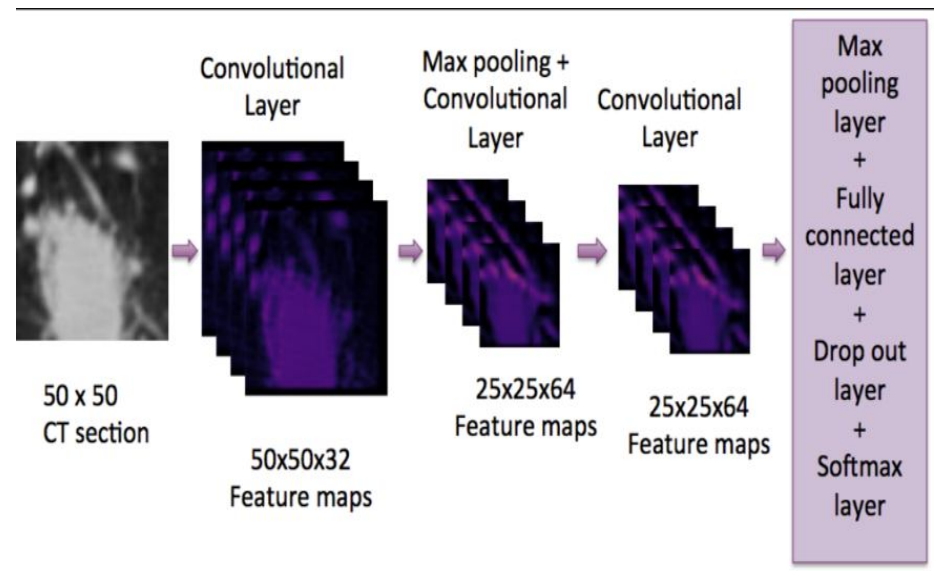

Figure 3: Proposed Architecture of CNN

We are prepared to construct a CNN in this. Since we've had a bit of dabbling with Tensor Flow, we resolved for anything pretty easy, which was too much work. We opted for tflearn.

\subsection{Training the Model}

The data needed to train a $\mathrm{CNN}$ in this is very high. Working out the batch model is often beneficial. Loading all training data into memory isn't always easy, because you need sufficient memory to handle it and the devices as well.

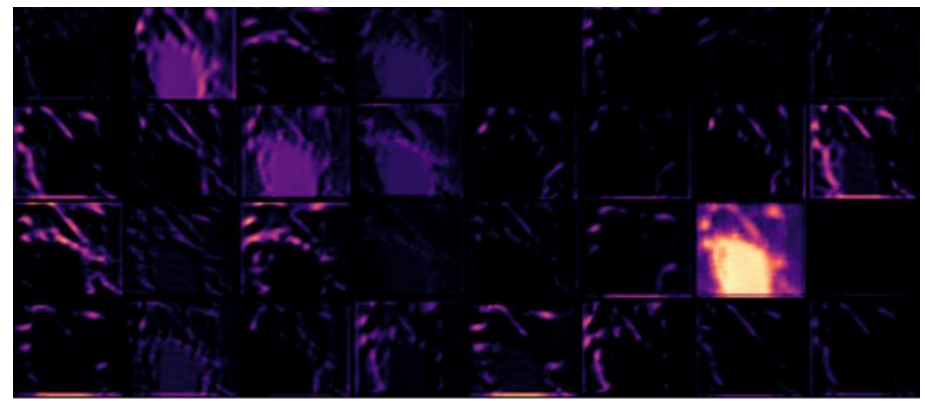

Figure 4: First convolution layer

Thus, we decided to use h5py library to load all images into a dataset of hdfs. Because as filters are of low resolution $(5 \times 5)$, it would have been more useful to visualize the maps of the created features. The characteristic map generated by the third convolutional layer with $643 \times 3$ filters is:

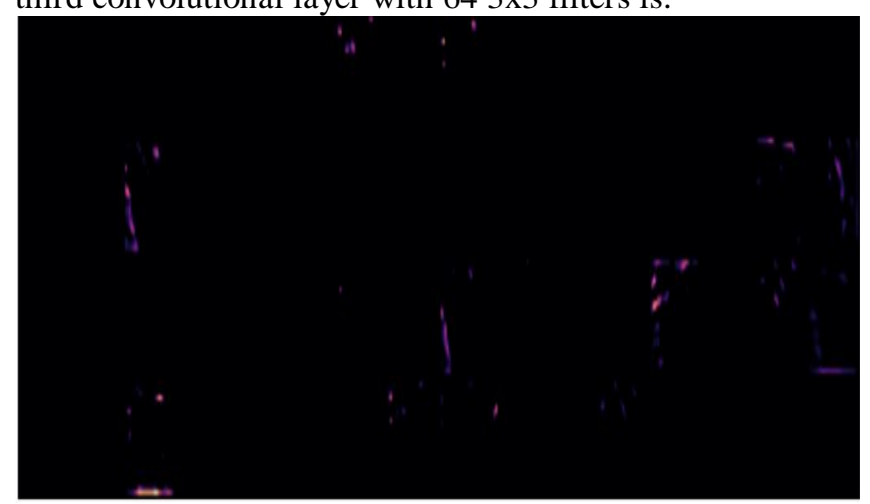

Figure 5: Feature Map generation 


\subsection{Testing the data}

For this we tested 1623 photos of our CNN platform. Then, we get 89 per cent validation accuracy. Our model has 84.3 percent accuracy and 68.2 percent recall. The model has an 89.2 per cent specificity.

\section{RESULTS AND DISCUSSION}

When we're doing all those tests using CNN in this. We got $\mathrm{CNN}$, it was their best concept or algorithm. We provide all the algorithms with the tests and performance analysis required for precision. Our architecture employed three stages of convolution.

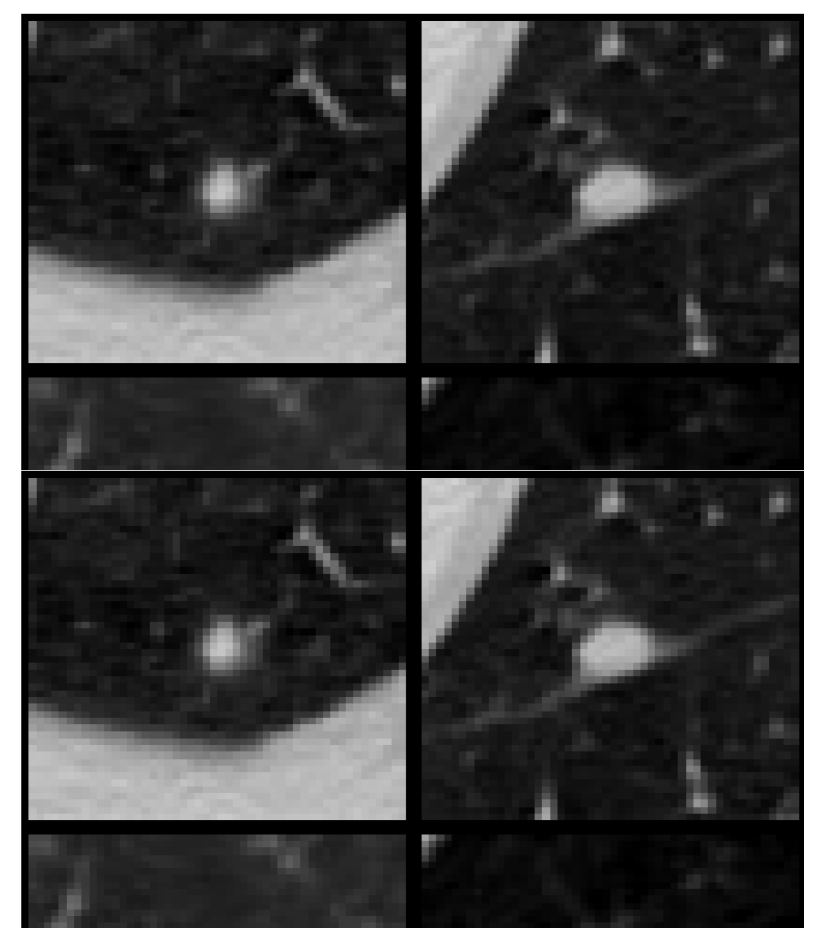

Figure 6: (a)Lung recognized Image 1

(b)Lung recognized Image 2

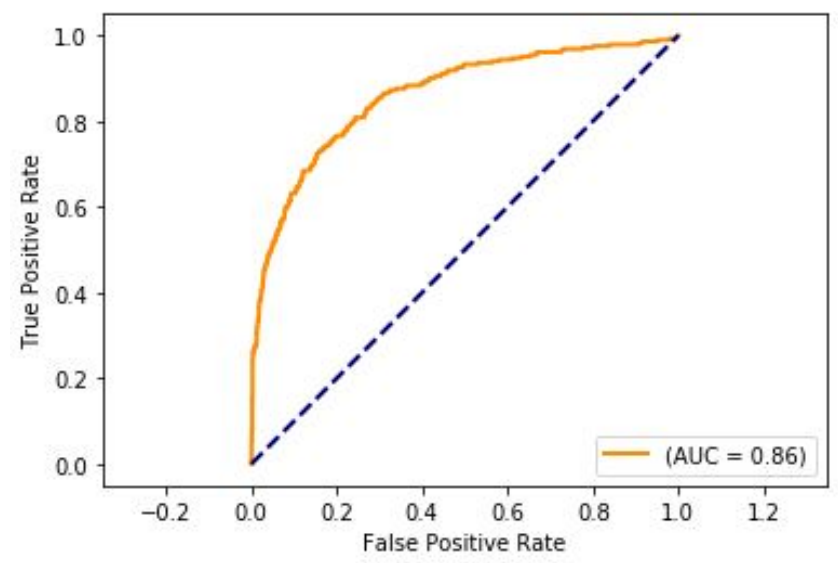

Figure 7: Performance prediction of True and Positive Predictions

\section{CONCLUSION}

The experimental results suggest that the techniques used in this approach have been adequately successful in segmenting the tumor in many images. Separately for each image they consider the intensity variance of the image. These were realized that perhaps the created framework would have the option of portioning tumors of different tumor and non-tumor images from the pre-owned database by achieving high precision. We get an 89 per cent approval accuracy. Our model is 89.3 percent accurate and 68.2 percent accurate. The model has an 89.2 per cent particularity. The structure proposed is kept an eye on JSRT informational collection and the discoveries have been seen as right. Later on, a more prominent number of pictures will be tried on our proposed framework and precision will be estimated. They ought to liken our work with existing works.

\section{REFERENCES}

1. Nardelli P, Jimenez-Carretero D, Bermejo-Pelaez D, Washko GR, Rahaghi FN, Ledesma-Carbayo MJ, Estépar RS. Pulmonary artery-vein classification in CT images using deep learning. IEEE transactions on medical imaging. 2018 May 4;37(11):2428-40. https://doi.org/10.1109/TMI.2018.2833385

2. Xie Y, Xia Y, Zhang J, Song Y, Feng D, Fulham M, Cai W. Knowledge-based collaborative deep learning for benign-malignant lung nodule classification on chest CT. IEEE transactions on medical imaging. 2018 Oct 17;38(4):991-1004. https://doi.org/10.1109/TMI.2018.2876510

3. Kumar A, Fulham M, Feng D, Kim J. Co-learning feature fusion maps from PET-CT images of lung cancer. IEEE Transactions on Medical Imaging. 2019 Jun 17;39(1):204-17.

4. Wang Q, Zhou X, Wang C, Liu Z, Huang J, Zhou Y, Li C, Zhuang H, Cheng JZ. WGAN-Based Synthetic Minority Over-Sampling Technique: Improving Semantic Fine-Grained Classification for Lung Nodules in CT Images. IEEE Access. 2019 Jan 30;7:18450-63.

5. Han T, Nunes VX, Souza LF, Marques AG, Silva IC, Junior MA, Sun J, Rebouças Filho PP. Internet of Medical Things-Based on Deep Learning Techniques for Segmentation of Lung and Stroke Regions in CT Scans. IEEE Access. 2020 Apr 14;8:71117-35.

6. Chung H, Ko H, Jeon SJ, Yoon KH, Lee J. Automatic lung segmentation with juxta-pleural nodule identification using active contour model and Bayesian approach. IEEE journal of translational engineering in health and medicine. 2018 May $18 ; 6: 1-3$. https://doi.org/10.1109/JTEHM.2018.2837901 
7. Li X, Dong X, Lian J, Zhang Y, Yu J. Knockoff filter-based feature selection for discrimination of non-small cell lung cancer in CT image. IET Image Processing. 2018 Dec 18;13(3):543-8.

8. Wang B, Si S, Cui E, Zhao H, Yang D, Dou S, Zhu J. A fast and efficient CAD system for improving the performance of malignancy level classification on lung nodules. IEEE Access. 2020 Feb 27;8:40151-70. https://doi.org/10.1109/ACCESS.2020.2976575

9. Devarapalli RM, Kalluri HK, Dondeti V. Lung Cancer Detection of CT Lung Images.

10. Gerard SE, Patton TJ, Christensen GE, Bayouth JE, Reinhardt JM. FissureNet: A deep learning approach for pulmonary fissure detection in CT images. IEEE transactions on medical imaging. 2018 Aug 10;38(1):156-66.

https://doi.org/10.1109/TMI.2018.2858202 